\title{
SHORT NOTE ON CONVEXITY OF POWER MEAN
}

\author{
LADISLAV MATEJÍČKA
}

\begin{abstract}
In this paper, we give an answer to the conjecture posed in the paper [Antal Bege, József Bukor, János T. Tóth, On (log-) convexity of power mean, Annales Mathematicae et Informaticae 42 (2013) pp. 3-7, http://ami.ektf.hu].
\end{abstract}

\section{Introduction}

Within the past years, the power mean has been the subject of intensive research. Many interesting results and inequalities for the power mean can be found in the literature. Mildorf (see [2]) studied the function $f(p, a)=M_{p}(1, a)=\left(\frac{1+a^{p}}{2}\right)^{\frac{1}{p}}$ and proved that for any given real number $a>0$ the following assertion hold:

(A) for $p \geq 1$ the function $f(p, a)$ is concave in $p$,

(B) for $p \leq-1$ the function $f(p, a)$ is convex in $p$.

In the paper (see [1]), authors studied the log-convexity and the log-concavity of power mean and proved:

Theorem 1. Let $f(p, a)=M_{p}(1, a)$. We have

$$
\begin{aligned}
& \text { for } p \leq 0 \text { the function } f(p, a) \text { is log-convex in } p \\
& \text { for } p \geq 0 \text { the function } f(p, a) \text { is log-concave in } p, \\
& \text { for } p \leq 0 \text { the function } f(p, a) \text { is convex in } p .
\end{aligned}
$$

Authors also have posted the following conjecture.

Conjecture. Let $M_{p}(a, b)$ be the power mean of order $p$ of two positive real numbers, $a$ and $b$, which is defined by

$$
M_{p}(a, b)=\left\{\begin{aligned}
\left(\frac{a^{p}+b^{p}}{2}\right)^{\frac{1}{p}}, & p \neq 0 \\
\sqrt{a b}, & p=0
\end{aligned}\right.
$$

Received December 08, 2014, accepted May 29, 2015.

2010 Mathematics Subject Classification. 26E60, 26D20.

Key words and phrases. Power mean, convexity, concavity. 
then

$$
\begin{aligned}
& \alpha=\inf _{a, b>0}\left\{p: M_{p}(a, b) \text { is concave for variable } p\right\}=\frac{\ln 2}{2}, \\
& \beta=\sup _{a, b>0}\left\{p: M_{p}(a, b) \text { is convex for variable } p\right\}=\frac{1}{2} .
\end{aligned}
$$

Our aim is to prove this conjecture.

\section{Solution of conjecture}

We prove (2),(3). Without loss of generality, we assume that $0<b<a$. Denote $s=\frac{b}{a}$. Suppose $p \neq 0$. We have

$$
M_{p}(a, b)=\left(\frac{a^{p}+b^{p}}{2}\right)^{\frac{1}{p}}=a M_{p}(1, s) .
$$

It is clear that $M_{p}(a, b)$ is concave (convex) function in $p$ for given $a, b>0, b<a$ if and only if $M_{p}(1, s)$ is concave (convex) function in $p$ for $s=\frac{b}{a}, 0<s<1$.

Some calculations give

$$
\begin{aligned}
\frac{\partial}{\partial p}\left(M_{p}(1, s)\right)= & M_{p}(1, s)\left[\frac{s^{p} \ln s}{p\left(1+s^{p}\right)}-\frac{1}{p^{2}} \ln \left(\frac{1+s^{p}}{2}\right)\right] \\
\frac{\partial^{2}}{\partial p^{2}}\left(M_{p}(1, s)\right)= & M_{p}(1, s)\left[\left(\frac{s^{p} \ln s}{p\left(1+s^{p}\right)}-\frac{1}{p^{2}} \ln \left(\frac{1+s^{p}}{2}\right)\right)^{2}\right. \\
& \left.+\frac{2}{p^{3}} \ln \left(\frac{1+s^{p}}{2}\right)-\frac{s^{p} \ln s}{p^{2}\left(1+s^{p}\right)}+\frac{s^{p} \ln s\left(p \ln s-1-s^{p}\right)}{p^{2}\left(1+s^{p}\right)^{2}}\right] .
\end{aligned}
$$

$\frac{\partial^{2}}{\partial p^{2}}\left(M_{p}(1, s)\right) \lessgtr 0$ is equivalent to

$$
\begin{aligned}
F(p, s)= & \left(\frac{s^{p} \ln s^{p}}{1+s^{p}}-\ln \left(\frac{1+s^{p}}{2}\right)\right)^{2} \\
& +p\left[2 \ln \left(\frac{1+s^{p}}{2}\right)-\frac{s^{p} \ln s^{p}}{1+s^{p}}+\frac{s^{p} \ln s^{p}}{\left(1+s^{p}\right)^{2}}\left(\ln s^{p}-1-s^{p}\right)\right] \lessgtr 0 .
\end{aligned}
$$

Put $G(p, t)=F\left(p, t^{\frac{1}{p}}\right)$ and

$$
g(t)=2 \ln \left(\frac{1+t}{2}\right)-\frac{t \ln t}{1+t}+\frac{t \ln t}{(1+t)^{2}}(\ln t-1-t),
$$

then $g(t)<0$ for $0<t<1$ and $t>1$.

Really, $g(1)=0$ and

$$
g^{\prime}(t)=\frac{(1-t) \ln ^{2}(t)}{(1+t)^{3}}
$$


implies that $g(t)<0$ for $0<t$ and $t \neq 1$.

From (1) we have

$$
\begin{aligned}
\inf _{0<s<1}\{p: F(p, s)<0\} & =\inf _{0<s<1}\{p>0: F(p, s)<0\} \\
& =\inf _{0<t<1}\{p>0: G(p, t)<0\}, \\
\sup _{0<s<1}\{p: F(p, s)>0\} & =\sup _{0<s<1}\{p>0: F(p, s)>0\} \\
& =\sup _{0<t<1}\{p>0: G(p, t)>0\} .
\end{aligned}
$$

From this we get $G(p, t) \lessgtr 0$ is equivalent to

$$
p \gtrless-\frac{h(t)}{g(t)}=\frac{\left(\frac{t \ln t}{1+t}-\ln \left(\frac{1+t}{2}\right)\right)^{2}}{-2 \ln \left(\frac{1+t}{2}\right)+\frac{t \ln t}{1+t}-\frac{t \ln t}{(1+t)^{2}}(\ln t-1-t)}=H(t) .
$$

We show that $H(t)$ is an increasing function on $(0,1)$. So, we obtain $\alpha=\lim _{t \rightarrow 0^{+}} H(t)$ and $\beta=$ $\lim _{t \rightarrow 1^{-}} H(1)$.

Now, we use the following Theorem 2 (see [3]).

Theorem 2. Let $f, g:[a, b] \rightarrow R$ be two continuous functions, which are differentiable on $(a, b)$. Further, let $g^{\prime} \neq 0$ on $(a, b)$. If $\frac{f^{\prime}}{g^{\prime}}$ is increasing (or decreasing) on $(a, b)$, then the functions $\frac{f(x)-f(b)}{g(x)-g(b)}$ and $\frac{f(x)-f(a)}{g(x)-g(a)}$ are also increasing (or decreasing) on $(a, b)$.

Because of $h(1)=0$ and $g(1)=0$ it suffices to show that $L(t)=-\frac{h^{\prime}(t)}{g^{\prime}(t)}$ is an increasing function on $(0,1)$. Some calculation gives

$$
L(t)=\frac{q(t)}{v(t)}=\frac{2\left(\frac{t \ln t}{1+t}-\ln \left(\frac{1+t}{2}\right)\right)}{\frac{(t-1) \ln t}{1+t}} .
$$

From $q(1)=0$ and $v(1)=0$ we have that it suffices to show $M(t)=\frac{q^{\prime}(t)}{v^{\prime}(t)}$ is an increasing function on $(0,1)$. Some calculation gives

$$
M(t)=\frac{w(t)}{r(t)}=\frac{2 \ln t}{2 \ln t+t-\frac{1}{t}} .
$$

Because of $w(1)=0$ and $r(1)=0$ it suffices to show that $Q(t)=\frac{w^{\prime}(t)}{r^{\prime}(t)}$ is an increasing function on $(0,1)$. Easy calculation get $Q(t)=\frac{2}{2+t+\frac{1}{t}}$. From $Q^{\prime}(t)=\frac{2\left(1-t^{2}\right)}{t^{2}\left(2+t+\frac{1}{t}\right)^{2}}>0$, we have $Q(t)$ is an increasing function, so $H(t)$ is also an increasing function. It implies

$$
\alpha=\lim _{t \rightarrow 0^{+}} H(t)=\frac{\ln 2}{2} \text { and } \beta=\lim _{t \rightarrow 1^{-}} H(t)=\lim _{t \rightarrow 1^{-}} Q(t)=\frac{1}{2} .
$$

The proof our conjecture is complete. 
Remarque. We note, that the results in the paper ([1]) can be obtained also by classical way. Using the same notation we have

$$
g(p, s)=\ln f(p, s)=\ln M_{p}(1, s)=\frac{1}{p} \ln \left(\frac{1+s^{p}}{2}\right)
$$

where $s=\frac{a}{b}, 0<b<a$.

Easy calculation gives

$$
\begin{aligned}
\frac{\partial}{\partial p} g(p, s) & =-\frac{1}{p^{2}} \ln \left(\frac{1+s^{p}}{2}\right)+\frac{1}{p} \frac{s^{p} \ln s}{1+s^{p}} \\
\frac{\partial^{2}}{\partial p^{2}} g(p, s) & =\frac{2}{p^{3}} \ln \left(\frac{1+s^{p}}{2}\right)-\frac{2}{p^{2}} \frac{s^{p} \ln s}{1+s^{p}}+\frac{1}{p} \frac{s^{p} \ln ^{2} s}{\left(1+s^{p}\right)^{2}} .
\end{aligned}
$$

Put $t=s^{p}$ then

$$
\frac{\partial^{2}}{\partial p^{2}} g(p, s)=\frac{1}{p^{3}}\left[2 \ln \left(\frac{1+t}{2}\right)-\frac{2 t \ln t}{1+t}+\frac{t \ln ^{2} t}{(1+t)^{2}}\right]=\frac{1}{p^{3}} k(t) .
$$

From $k(1)=0$ and from

$$
k^{\prime}(t)=\frac{2}{1+t}-2 \frac{1+t+\ln t}{(1+t)^{2}}+\frac{\ln t((1-t) \ln t+2(1+t))}{(1+t)^{3}}=\frac{(1-t) \ln ^{2} t}{(1+t)^{3}}
$$

we have $k(t)<0$ for $t>0$ and $t \neq 1$. It implies the results of ([1]).

Open Problem. It is evident that for $p \leq 0$ the function $M_{p}(1, a), a>0$, is also exponentially convex for variable $p$. But, what are equal to

$$
\begin{aligned}
& \inf _{a, b>0}\left\{p: M_{p}(a, b) \text { is exponentially concave for variable } p\right\}, \\
& \sup _{a, b>0}\left\{p: M_{p}(a, b) \text { is exponentially convex for variable } p\right\} .
\end{aligned}
$$

\section{References}

[1] Antal Bege, József Bukor, János T. Tóth, On (log-) convexity of power mean, Annales Mathematicae et Informaticae, 42 (2013), 3-7, Available online at http: / / ami . ektf . hu.

[2] T. J. Mildorf, A sharp bound on the two variable power mean, Mathematicals Reflections, 2 (2006), 5 pages, Available online at http: / /awesomemath .org./wp-content/uploads/reflections/2006-2/2006-2-sharpbound.pdf.

[3] I. Pinelis, L'Hospital type results for monotonicity, with applications, Journal of Inequalities in Pure and Applied Mathematics, 3 (2002), 1-5.

Nálepkova 237, 01901 Ilava, Slovakia.

E-mail: ladislav.matejicka@tnuni.sk 